\title{
Utilizing the Affinity Research Group Model in a Summer Research Experience for Undergraduates Program
}

\author{
Ben Jelen \\ Indiana University \\ Bloomington, Indiana \\ bcjelen@indiana.edu
}

\author{
Julia Dunbar \\ University of Washington \\ Seattle, Washington \\ jcd17@uw.edu
}

\author{
Susan Monsey \\ Indiana University \\ Bloomington, Indiana \\ skmonsey@iu.edu
}

\author{
Olivia K. Richards \\ Pennsylvania State University \\ University Park, Pennsylvania \\ orichards@psu.edu
}

\author{
Katie A. Siek \\ Indiana University \\ Bloomington, Indiana \\ ksiek@indiana.edu
}

\begin{abstract}
In this paper, we describe how we integrated the Affinity Research Group (ARG) model into a 10-week summer research experience for undergraduates (REU) over the last three years. Each summer, ten REU students participated in: an annual two-day team-building orientation; a four day long ramp-up session to learn about REU projects and the skill sets they would need to master; weekly meetings with graduate and faculty mentors to define objectives, deliverables, and progress; and weekly skill development workshops to learn how to conduct research, prepare for future endeavors, and present oneself in professional settings. A total of seven faculty and twelve graduate students participated in ARG model process meetings to ensure students received comparable experiences and facilitated professional development activities. We provide an overview of our REU ARG model experience and reflect on improvements based on faculty mentor, graduate mentor, and undergraduate researchers' experiences.
\end{abstract}

\section{CCS CONCEPTS}

- Social and professional topics $\rightarrow$ Computing education; Computing education programs; • Applied computing $\rightarrow$ Health informatics;

\section{KEYWORDS}

Research Experience for Undergraduates (REU); Affinity Research Group (ARG) model; mentoring

\section{ACM Reference Format:}

Ben Jelen, Julia Dunbar, Susan Monsey, Olivia K. Richards, and Katie A. Siek. 2019. Utilizing the Affinity Research Group Model in a Summer Research Experience for Undergraduates Program. In Proceedings of the 50th ACM Technical Symposium on Computer Science Education (SIGCSE '19), February 27-March 2, 2019, Minneapolis, MN, USA. ACM, New York, NY, USA, 7 pages. https://doi.org/10.1145/3287324.3287501

Permission to make digital or hard copies of all or part of this work for personal or classroom use is granted without fee provided that copies are not made or distributed for profit or commercial advantage and that copies bear this notice and the full citation on the first page. Copyrights for components of this work owned by others than the author(s) must be honored. Abstracting with credit is permitted. To copy otherwise, or republish, to post on servers or to redistribute to lists, requires prior specific permission and/or a fee. Request permissions from permissions@acm.org.

SIGCSE '19, February 27-March 2, 2019, Minneapolis, MN, USA

( 2019 Copyright held by the owner/author(s). Publication rights licensed to ACM. ACM ISBN 978-1-4503-5890-3/19/02 ..\$15.00

https://doi.org/10.1145/3287324.3287501

\section{INTRODUCTION}

Research experiences for undergraduates (REUs) are valuable from a student, faculty, and university perspective [23]. More recently, REUs have been recognized as a valuable graduate student opportunity $[1,11]$. REUs can improve undergraduate retention in STEM - especially in underrepresented and first generation college student groups $[4,13]$. Undergraduate researchers learn about critical thinking, communication skills, grit, and comfort with uncertainty $[23,29]$ - although the uncertainty must be appropriately scaffolded within a well planned research experience $[8,30]$. Faculty can improve their teaching, mentoring, and provide applied learning experiences to students while building on their own research agendas [23] by iteratively collaborating with graduate student mentors to provide a personalized undergraduate research experience [30]. In addition, faculty learn to mentor students from diverse backgrounds while possibly addressing their own biases [4] if gender equity programming is available [14]. Graduate students, who are being trained as near-future scholars and major contributors to scientific knowledge, are not well investigated in their role as REU mentors [1] - despite faculty reporting that they learned on their own how to mentor researchers [4]. Finally, universities improve scientific contributions [23], student engagement [4], and community outreach through REUs.

Undergraduate researcher experiences are dependent on how the undergraduate is immersed in an organized program with a developed social, scholarly group culture $[4,8]$. In addition, undergraduate researchers must feel they are contributing in a well scaffolded research project that will make contributions to a scientific community [4] while also aligning with their cultural, contextual, political, and desires to serve the broader community [15, 29]. In this experience report, we contribute: (1) an overview of how the Affinity Research Group (ARG) model was applied to Indiana University's 10-week summer ProHealth REU site and (2) qualitative experiences of undergraduate researchers, faculty mentors [4], and the less studied, but integral graduate student mentor perspectives.

The ProHealth REU site provided a diverse cohort of 30 undergraduate scholars with opportunities to contribute to health and computing communities through research dissemination and continue on in their academic pursuits with dedicated mentorship by seven faculty and twelve graduate student mentors. 


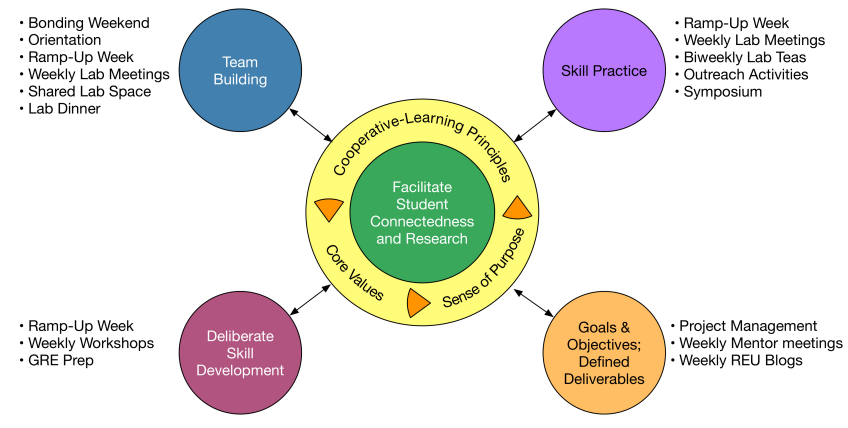

Figure 1: Affinity Research Group Model elements adapted from $[8,32]$ with our REU site activities listed near each element.

\section{BACKGROUND}

Since the ProHealth REU site engages undergraduates in interdisciplinary research, we aimed to extend project-based learning, which has been successfully applied to interdisciplinary undergraduate research endeavors [9], so that students could transition from practitioner to apprentice to researcher. Dahlberg et al. successfully applied situated learning theory to fully immerse undergraduates in a community of practice and integrate them into a research community [6], however we were unsure how to integrate the theory. Villa et al. mapped how situated learning theory elements relate to ARG model experiences [32] which has been shown to successfully develop a cooperative computing research experience [8, 31, 32].

Thus, we we utilized the Affinity Research Group model where faculty and graduate student mentors share their affinity for their research to undergraduate students. The mentors guide undergraduate researchers in developing new skills so that each research team member - including the undergraduate researcher with a diverse background - becomes a subject-matter expert. With their new expertise, undergraduate researchers become equal contributors to the research group [8]. The ARG model is composed of four main elements: team building; deliberate skill development; skill practice; and goals and objectives with accompanying defined deliverables [32]. These elements are often accompanied with close mentorstudent interactions, professional development, and communication skills. When all of these elements become routine, they become part of the lab culture that empowers research team members to implement what they have learned into research progress [8].

\section{REU SITE OVERVIEW}

The main intellectual pursuit for the ProHealth REU site at Indiana University was to develop undergraduates from diverse backgrounds into scholars who can contribute to the design, development, and evaluation of pervasive systems that empower lay people to proactively manage their health and wellness. Students were fully engaged in cross-cutting computing research that can solve real world health problems and make an impact on people's lives - two factors that have been shown to attract underrepresented groups in computing $[19,20]$. The ProHealth REU provided students with a unique opportunity to learn techniques in the areas of human computer interaction (HCI), health informatics, pervasive computing (mobile or wearable), environmental sensing (ambient and on-body), machine learning, and the social and legal implications of these technologies.

\subsection{Administration}

Our REU site was led by a principal investigator (PI) who coordinated with a school-level assistant dean and external evaluator. The assistant dean assisted with recruitment, coordinating with university-level undergraduate research summer programs, and logistics including student travel, social activities, orientation, room and board, and university-level accounts (e.g., building access cards, stipend payments, and email). The experienced external evaluator assessed the REU site by facilitating interviews at the start and end of the REU, administering the CISE Common REU Evaluation Toolkit [28], administering site-specific surveys pre, mid, and post experience, and coordinating with our service-learning collaborators to assess the impact of the REU site's service activities. The PI also recruited and trained faculty and graduate student mentors, recruited and coordinated training for the undergraduate researchers, and followed up with all REU site stakeholders. Faculty and graduate student mentors utilized materials from the ARG model $[8,31,32]$ to become effective mentors who engendered a supportive, collaborative research community that provided students with diverse abilities the opportunity to learn research skills while making scientific, research contributions.

\subsection{Site Demographics}

Since 2016, the REU site has trained and funded 30 undergraduate researchers, trained 10 research group affiliated undergraduate researchers funded through federal and internal programs, and trained 47 school-affiliated undergraduate researchers who were funded similarly. All affiliated undergraduate researchers participated in the same training workshops led by our REU site mentors and social experiences led by the assistant dean. Thus, our REU site benefited our school by providing training, social, and administrative programming resources.

The ProHealth REU site was committed to diversifying computing research experiences for traditionally underserved groups where $87 \%$ (26/30) of participants were from groups traditionally underrepresented in computing (73\% (22/30) women, 37\% (11/30) underrepresented minority (URM) groups, and 33\% (10/30) first generation college students). The ProHealth REU site's participants were more diverse than 30 REU sites reported in 2016 where $42 \%$ were women and $34 \%$ were from URM groups [29]. In the ProHealth REU, 56\% (17/30) were from liberal arts or commuter universities.

\subsection{Experience}

The ProHealth REU site experience immersed five pairs of undergraduates in a 10-week research community with collective mentoring from graduate students and faculty following the ARG model as shown in Figure 1 with accompanying activities in Table 1 . In this section, we bolded ARG model elements and italicized our $R E U$ site activities that fulfilled these elements.

Each summer, we kicked off the REU site with a leadership and team development weekend for team building at a local adventure education facility. After the bonding weekend, undergraduate 


\begin{tabular}{l|l|l} 
W & Activities & Deliverables \\
\hline 1 & $\begin{array}{l}\text { Leadership and Team Dev. Bonding Weekend(TB); Orientation(TB); Ramp-up Week } \\
\text { sessions(TB, SD, SP); ARG review; Tea: Elevator Pitches(SP); LM(TB, SP) }\end{array}$ & Ramp-up Assignments; Blog posts \\
\hline 2 & WW: Reading papers and documenting(SD); LM(TB, SP) & Paper: Related Work and Methods outlines; Blog post \\
\hline 3 & $\begin{array}{l}\text { WW: Writing Research Papers(SD); Tea: Improv Communication(SP);Mentor Update } \\
\text { Meeting; GRE Prep(SD); LM(TB, SP) }\end{array}$ & Paper: Related Work and Methods drafted and Introduction outline; Blog post \\
\hline 4 & WW: Online Presence(SD); Outreach Activity(SP); GRE Prep(SD); LM(TB) & $\begin{array}{l}\text { Paper: Related Work and Methods peer review, Introduction draft, Abstract } \\
\text { outline; Web Presence: drafted; Blog post }\end{array}$ \\
\hline 5 & $\begin{array}{l}\text { WW: Graduate Admissions(SD); Tea: Inclusion and Diversity(SP); Lab dinner(TB); GRE } \\
\text { Prep(SD); LM(TB, SP) }\end{array}$ & $\begin{array}{l}\text { Paper: Related Work and Methods final, Introduction peer review, Abstract draft, } \\
\text { Findings and Discussion outlines; Web presence: Iterate on draft; Blog post }\end{array}$ \\
\hline 6 & $\begin{array}{l}\text { WW: Presenting Research(SD); Evaluator Update to Mentors; GRE Prep(SD); LM(TB, } \\
\text { SP) }\end{array}$ & $\begin{array}{l}\text { Paper: Introduction draft, Abstract peer review, Findings and Discussion drafts; } \\
\text { Web Presence: final; Lightning Talk: draft; Blog post }\end{array}$ \\
\hline 7 & $\begin{array}{l}\text { WW: Networking and Imposter Syndrome(SD); Tea: Lightning Talks(SP); Outreach } \\
\text { Activity(SP); GRE Prep(SD); LM(TB, SP) }\end{array}$ & $\begin{array}{l}\text { Paper: Introduction final, Abstract draft, Findings and Discussion peer reviews; } \\
\text { Project Video: storyboard; Poster: sketch; Blog post }\end{array}$ \\
\hline 8 & WW: Poster Review(SD, SP); GRE Prep(SD); LM(TB, SP) & $\begin{array}{l}\text { Paper: Abstract peer review, Findings and Discussion drafts; Project Video: draft; } \\
\text { Poster: draft; Blog post }\end{array}$ \\
\hline 9 & WW: Researching Remotely(SD); Tea: Future Plans(SD); GRE Prep(SD); LM(TB, SP) & $\begin{array}{l}\text { Paper: Abstract final, Findings and Discussion final; Project Video: final; Poster: } \\
\text { iterate on draft; Blog post }\end{array}$ \\
\hline 10 & WW: Industry Research Careers(SD); Poster Symposium(SP); Mentor debrief & Paper: final; Web Presence: final; Poster: final; Blog post \\
\hline
\end{tabular}

Table 1: Week by week schedule of our REU site. The ARG activity categories are team building (TB), skill development (SD), and skill practice (SP). The fourth ARG category - deliverables - is separated out into its own column. WW indicates weekly workshop professional development topics. LM indicates weekly lab meetings.

researchers moved into a shared living space. Mentors regularly met in preparation for the REU by iteratively developing research summaries for students based on ARG model activities [31]. Summaries included research project goals, student profile, tasks, deliverables, and benefits for the student, researchers, and research community.

For undergraduates, the first week started with orientation where they learned about the university and research group before starting ramp-up week. Since students were from diverse backgrounds with varying skill sets and research experience, undergraduates were not assigned to research projects before arriving. Instead, we gave them a glimpse into the different projects and skill sets they would master for each project during a week-long intensive session. This "ramp-up week" gave students the opportunity to decide if they wanted to dive deeper into statistics to be proficient at machine learning or work with older adults in a user study. Rampup week consisted of short, 30-90 minute sessions that provided students with basic research training (e.g., ethics training, finding related work), lab expectations, program aims, short academic presentations highlighting research projects, and project-specific skill training workshops. Students received short assignments to practice skills each night and blog about. Meanwhile, faculty and graduate student mentors taught the workshops and had an opportunity to work with the undergraduate researchers. At the end of the week, the undergraduates submitted their top three research project choices. Mentors met to assign undergraduates in pairs to projects based on their preferences and how the undergraduates did on their assignments. The week culminated in our first of five lab working teas where undergraduates gave elevator pitches about their research project.

Undergraduates worked together in a shared lab space near the faculty and graduate student mentors' offices, but with the flexibility to work in reserved breakout rooms or nearby research smart homes. Faculty and graduate student mentors collaboratively worked with REU students in at least weekly meetings on project management to define research goals, outline specific tasks, and develop a timeline that was iterated on throughout the summer. Students became familiar with the project through scholarly readings, continued tutorials, and guided task-based learning during the first few weeks.
Once students developed a deeper understanding of the problem space and began to master skills, students continued working on their research tasks to help them mature into independent scholars who contributed to the larger projects. Mentors coordinated with interested undergraduates on how to continue the research at their home institution through independent studies after the REU.

Undergraduates developed skills at mentor-taught weekly workshops where students learned research and professional development skills. Weekly workshops were scheduled so that students learned each skill within a week of applying it to their deliverables.

Research teams created a mix of deliverables that provided students the opportunity to practice their professional oral, written, and multi-media-based communication skills. Students iteratively wrote a conference-quality paper. The papers were peerreviewed by mentors and fellow undergraduate researchers so that undergraduates not only received feedback on their own paper, but they could learn from their colleagues' papers. Students developed a professional web presence via a website with a C.V., social media, and video that summarized their research contributions. Students learned to professionally present their research via presentations at weekly lab meetings and lab teas and posters at an end-of-summer symposium. Students documented their experience in their weekly blog posts to reflect on how they developed as a researcher and to assist the mentors and leadership team identify how to better support the developing scholars.

Undergraduates and mentors practiced their computing skills and gave back to the public by leading two two-hour long outreach activities during the summer. Activities included teaching middle school and high school campers and older adults at an adult-education event about circuitry via a paper circuit activity [25].

\subsection{Site Results}

The ProHealth REU site external evaluator administered pre- and post-program Computer and Informatics Sciences and Engineering (CISE) REU Toolkit assessments for the purpose of evaluating the effectiveness of the CISE REU program [28]. The evaluation team matched most of the REU site's pre-program and post-program assessment data (2016, N=9; 2017, N=8) based on those who provided 
identifying information and then ran a non-parametric Wilcoxon analysis test to assess for significant differences among assessment categories. ProHealth REUs improvement in research selfefficacy $(M=4.56)$ was statistically significant than pre-program values $(M=3.82), Z=-3.30, p=.001$. In addition, the mean post-program values for Research Skills $(M=4.412)$ were statistically significantly higher than mean pre-program values $(M=3.39), Z=-2.55, p=.011$. ProHealth REUs had better mean gains than the 2017 cohort of 38 REU sites in research self-efficacy, grit, and leadership [26]. During pre- and post-interviews with ProHealth REUs, we found that their experience helped them: (1) learn about new fields in computing that they otherwise had not been exposed to (e.g., HCI, machine learning, and health informatics); (2) mature in their understanding of research from a "solve a problem" mentality to research as a collaborative, iterative process; and (3) become enthusiastic about conducting research to improve the lives of others.

We surveyed mentors ${ }^{1}$ over the 3 years $(\mathrm{N}=27)$ to assess the load of advising REUs and mutual benefits since our program has engaged four junior faculty to lead six projects during the first three years. During a "normal week," most mentors spent over 3 hours advising REU students and 1-2 hours prepping for these meetings. Faculty mentors spent 1-2 hours a week mentoring graduate students on how to advise undergraduates. All mentors said that their time was well spent and $60 \%(\mathrm{~N}=16)$ of mentors thought that their research with REUs would result in a publication. All mentors taught students skills and engaged socially with REUs (e.g., lunch).

Seven out of eight REUs who have graduated have gone on to graduate school - twenty-two are still working on their undergraduate degrees in various fields - from computer science to mathematics to health policy and political science. Of those still working on their degrees, nine reported obtaining research experiences in subsequent summers. One REU completed her MS and recently started a PhD Program - for a total of four REU students currently enrolled in $\mathrm{PhD}$ programs.

Site REUs and research group affiliated REUs have contributed to seven full conference papers [3, 10, 12, 16, 18, 22, 24] - including 3 highly competitive conferences $[3,16,18]$ and a best paper award [24]. In addition, they have presented 3 workshop papers $[2,7,21]$ and placed in the ACM Undergraduate Research Competition at SIGCHI [5, 27]).

\section{EXPERIENCES}

We briefly provide stakeholder insights from our REU site to understand their roles and challenges they faced.

\subsection{Faculty Experiences - PI to Mentor}

As the Principle Investigator (PI), I was responsible for coordinating all of the site experiences in Table1 and working closely with faculty, graduate students, administrators, staff, external collaborators, and the undergraduate researchers.

I started planning the following year's REU site in the spring a few weeks before the current year's REU participants arrived to secure housing and team building activities. In the late summer and early fall, we recruited and interviewed undergraduates. In the fall, I followed-up with all REU stakeholders to reflect on the past

\footnotetext{
${ }^{1}$ mentors may serve multiple years
}

REU site and their current academic plans. These follow-ups often prompt mentors to reach out to the undergraduate researchers thus continuing their mentoring relationships. In the winter, I finalized our REU site participants and worked with administrators, staff, and the evaluator on university-level logistics. In late winter, I coordinated with faculty and graduate student mentors to identify who had a research project that could benefit their own research and undergraduate researchers. After we identified projects, we started our ARG model informed mentor training meetings. In the spring, I iterated on the schedule and recruited mentors to teach workshops and host teas throughout the 10-weeks to fulfill each element of the ARG model. During the REU site, I was the point person for anything related to our school's summer undergraduate research program. Throughout the year, I answered questions from REU participants and alumni; connected undergraduates with researchers in areas of interest; and coordinated travel for them to present their REU site research at conferences.

As a faculty mentor, my aim was to support the success of the undergraduate researchers and graduate student mentors. I worked with graduate student mentors to compartmentalize and identify tasks for the undergraduates. We typically iterated on the projects, research questions, tasks, and skills in the winter knowing that we most likely would iterate again once we saw the undergraduates' progress during ramp-up week. During weekly meetings with graduate mentors, we discussed how to keep students motivated for the parts of research that may not appeal to them, how authorship should be addressed, and how to adapt the project and time line based on current progress. I saw how these conversations translated into our research mentoring relationship - especially with respect to their dissertation progress.

I met weekly with undergraduate researchers. In most cases, the undergraduates we collaborated with met with a mentor 3-5 times a week. The graduate student mentors focused on teaching technical skills, whereas I worked on professional skills. We included undergraduate researchers in our collaborative meetings with researchers across the university and world. In each meeting, we ensured undergraduates could make contributions. Early in the summer, students introduced themselves and were prepared to ask about what related work and methods they should be researching. These interactions provided students with the opportunity to learn from other research collaborators. As the summer progressed, undergraduate researchers were responsible for presenting parts of the research and, by the end of the summer, showed confidence as they led longer discussions about their work.

Near the end of the REU, graduate student mentors and I worked with undergraduate collaborators to identify publication venues and discuss how we could continue supporting each other. In most cases, we decided to collaborate on a publication due within the next year and follow-up with each other about funding and collaboration opportunities. Indeed, two projects from this REU site were integral in showing the prior work necessary for a federal agency and internal university group to fund more of my research in this area.

\subsection{Graduate Mentor Perspective}

I was a Ph.D. student mentor in the REU for all three years. Prior to the start of each 10-week REU site, I met with my Ph.D. advisor to 
develop long-term goals for the summer. We discussed how to make the summer valuable for both my research and the REU students, including how to compartmentalize tasks to give the students a chance to grow their research skills. I met weekly with my advisor to discuss the students' progress and ask for advice as a PhD student interested in mentoring students as a future faculty member.

During the ramp-up session, I participated as a knowledge area expert and mentor. I presented on topics that were relevant to that years' projects, such as hardware with Arduino microcontrollers and finding related work. I presented my research to give students an idea of what they would work on if they chose to work with us.

I regularly met with my REU students - typically every other day - to answer questions and support them as they progressed. We started meetings with a recap of what they had accomplished since the last meeting and how to address any roadblocks they faced. I also summarized what I was working on that week to complement their efforts. I explicitly included time to talk about graduate studies, such as, "What should I include in my graduate school applications?", or "Should I aim for an M.S. or a Ph.D.?" We concluded with setting new goals for the next time we met. New goals included different tasks, so in case they ran into a roadblock, they could continue making progress on another task. Later in the REU, I encouraged mentees to choose their own goals to foster independence. I also check-in daily, informally with mentees. Occasionally we ate lunch as another opportunity for less formal interaction.

In addition to supporting the REU students I directly mentored, I supported the REU site's students. I joined the biweekly lab teas to contribute to the group culture. I met with several REU pairs when they were working on aspects of their projects where I am an expert. For example, I met with a pair to talk about running a design workshop with older adults. I also helped other students by connecting them to graduate students and prior REUs who work in their interest area, so they could get a feel for what graduate studies in that area might be like.

Lastly, I continued mentoring previous students long after the REU experience. I exchanged several emails with REU students checking in with them, offering advice, or coordinating a meeting at an upcoming conference. For both pairs I directly mentored in 2016 and 2017, I also worked with them on submitting papers based on the work we did together (e.g., [12]).

Reflecting on my experience, our REU informed by the ARG model made both practical progress on my research as well as developed my mentoring skills. The REU students helped move my dissertation research forward in one area, while I concurrently worked on another. Their help was valuable in completing a project in a short amount of time and turning it into a publication submission. As a $\mathrm{PhD}$ student interested in contributing to academia, I valued the opportunity to develop my mentoring skills. I also enjoyed that I was supporting underrepresented groups in computing to build up their research skills and become contributing members of an inclusive research community.

\subsection{Undergraduate Researcher Experience}

4.3.1 Year 1: 1st Year Undergraduate Experience. As a freshman from an R1 university majoring in math with little knowledge of informatics, but excitement as to what technology can do for good, the REU faculty gave me a chance as a participant in the 2016 REU program. That chance turned into an international research award, numerous poster presentations, graduate school preview weekends, and several other research experiences. The doors that my REU experience has opened for me and other bright undergraduate researchers, is inspiring.

I never thought I would have had the opportunity to attend a major ACM conference as a sophomore undergraduate, let alone publish my work as part of the Student Research Competition [27]. Without the foundation of research skills that I learned as part of the REU, it simply would not have been possible. Encouragement and support from my faculty and graduate mentors solidified my interest in pursuing a Ph.D. in human-computer interaction and helped to prepare me to excel as an independent researcher.

The REU created an atmosphere that I will strive to create in my own lab, one day. Our weekly lab social meetings and workshops provided the skills necessary to navigate professional and social dynamics within a research lab. The gatherings and daily routine created a culture of camaraderie. At times throughout the first few weeks of the REU, I was lost, but I learned to fail on my own often, rely on peers, and search for answers. The group culture taught me that I could ask anyone in the larger lab group for support. Through constant iteration working on my electronically enhanced textile prototype, I failed enough and learned enough to be the lab expert in e-textiles and sewing.

Educational workshops on topics from Tableau to qualitative research methods and the inherent structure of my REU experience taught foundational skills needed to develop my own research questions and craft a plan to realistically achieve my goals over ten short weeks. As a result of thriving with the structured deliverables of the REU, I also became skilled at creating independent research plans with goals and deadlines that I applied successfully to two large projects at the University of Washington, this past summer, as a CRA-W DREU student.

As a result of my REU experience, I gained a much deeper understanding of what research truly means as well as the diverse avenues that I can take to combine my varied interests of math, human-computer interaction, and innovation - which I did not know was possible. Our mentors' genuine combination of passion, connections, and expertise allowed our "basic undergraduate ideas" to flourish. The REU showed me the power that I have to work with others to make a difference in the STEM community.

4.3.2 Year 2: Senior Undergraduate Experience. Going into the summer 2017 REU, I was a rising senior who was unsure about what she wanted to do with her life. Coming out of the REU I was a rising senior who had a plan to apply straight into $\mathrm{PhD}$ programs related to the fields of Health Informatics and Human Computer Interaction (HCI). This REU made me fall in love with two fields, that quite frankly I did not even know existed until I did this REU.

Beyond discovering my next steps, I also learned a multitude of other lessons from this REU experience, such as current research skills related to my field, what to expect while completing my $\mathrm{PhD}$, and how to maintain a good work/life balance. During my experience I had the opportunity to be published alongside my mentors $[7,18]$, establishing myself as a researcher and expert regarding the interweaving and connections throughout the Asynchronous 
Remote Communities [17] method that my graduate mentor was just learning about. I learned a lot from both my mentors and also from the other students participating in the REU. It was great to be surrounded by students from different backgrounds that were equally as motivated as me about research.

I can go on and on about my good experiences from this REU, but there were also a few things that I wish would have been brought more into the experience as well. Such as, more feedback from my summer mentors (specifically about writing), time scheduled to talk to any mentor in or outside of the lab, and showing more of the difference and benefits of both academia and industry.

The overall experience was very hands on and for a beginning researcher, I found this to be mostly to my benefit. However, knowing how a $\mathrm{PhD}$ is very independent and you have to find a lot of self-motivation, I think at times there could have been more independence during the research process. Especially, creating our own goals and writing schedule. In comparison, these are small add-ons to my net gain from the REU experience. During this REU, I not only gained knowledge, but also a strong working relationship with the lab that I hope to keep in the future, because without this experience I would not be where I am today. Which is about to embark on my $\mathrm{PhD}$ at the University of Washington.

4.3.3 Year 3: Non-traditional Student Experience. I came to the REU with a different perspective than the rest of my undergraduate counterparts in the summer of 2018. As a non-traditional student in the midst of a career change (student affairs to computer science), I was looking to the REU as an opportunity to start re-building my resume and to expand my professional network. The program certainly met my goals.

As a second degree student, the REU was not my first experience with research, as I was heavily involved in research in the social sciences at my previous university. However, the REU introduced me to the field of Informatics and broadened my research skills in relation to computing, technology, and data analysis. A wonderful part of the REU research experience was the interaction with the rest of the research team, whether it was my fellow undergraduate researchers, the graduate student mentors, or the faculty mentors. While there was certainly several distinct research projects going on, everyone seemed willing to help out and share their own particular skills and expertise. This was particularly helpful to me, as the graduate student that was scheduled to serve as my mentor had to cancel at the last minute and was not replaced. While the lack of a graduate student mentor was certainly a frustration at times, in retrospect, it may have ended up being a blessing in disguise. I was able to develop a sense of independence in my research that might not have been possible otherwise.

An interesting aspect of the research that I was involved in was the fact that it was the pilot study for a brand-new research project with a new faculty member. Starting from ground zero, writing a portion of the IRB, and having the opportunity to have a significant voice in the selection of the equipment used for the study helped me become an expert in many aspects of environmental sensing.

The communication and research skills taught by our mentors during the program are some of the most valuable assets of the REU program. Learning how to manage the writing, editing, and peer review process for academic research papers will be helpful as I pursue my graduate studies. While the "deliverables" expected by the end of the 10-week REU seemed daunting at the outset, the faculty encouraged us to reach higher than we might have attempted without their encouragement.

\section{REFLECTIONS AND FUTURE ITERATIONS}

We utilized the ARG model based on past successes for creating a research experience in computing for undergraduates [8, 32]. Fortunately, the ARG model provided us with a base to conceptualize and implement our REU site. The undergraduate experiences noted here and in our evaluation reports discuss how undergraduates felt they were part of a research team (team building) and learned skills that they ordinarily would not have acquired in their academic programs (skill development and practice). They also noted how, although stressful at times, the deliverables prepared them to make contributions to science. In addition, faculty and graduate students appreciated the structured materials we adapted [8,32].

In terms of what worked, our REU site undergraduates developed a strong sense of community - which we plan to study more to see if it is ARG related, research area, or the diverse, inclusive cohort that was selected. Students appreciated being introduced to informatics and other areas of computing that, although recognized by the ACM, are not in all computing degree programs. The rampup week was beneficial for all stakeholders - undergraduates were able to make informed decisions on what projects they wanted to do; graduate students practiced teaching and mentoring; and faculty optimized their time with respect to teaching about their research and shared responsibility for research skill training by teaching 1-2 workshops instead of teaching all of the research skills to each undergraduate. Graduate student mentors were integral in the successful experiences of the undergraduates because there was always someone who was passionate about their research project.

We plan to iterate on two key areas - mentoring and schedule flexibility. Undergraduate researchers appreciated having a pair of mentors, however sometimes mentors travel (undergraduate experience in year 2) or cannot come because of emergencies (undergraduate experience in year 3). We will explore having a "contingency mentor" for each project where in addition to the faculty-graduate mentor pair, the contingency mentor will be aware of the research and available for events when other mentors cannot be reached. Since undergraduates present at weekly meetings and teas, the contingency mentor should be able to quickly learn about project updates and advise students. Although in most cases, we provided personalized mentoring [30], we need to work on personalizing our schedule in Table 1 to accommodate typical research roadblocks (e.g., electronic parts come in late) and assist in helping undergraduate researchers develop into independent scholars. We will investigate having a weekly workshop in week 4 , after students have drafted the methods section of their research paper, to discuss how to adapt the schedule and deliverables. We can revisit this schedule at each weekly workshop.

\section{ACKNOWLEDGEMENTS}

The REU site is supported by the National Science Foundation Award CNS-1560276. Ben Jelen was supported by National Science Foundation Award DGE-1342962. 


\section{REFERENCES}

[1] Benjamin Ahn and Monica F. Cox. 2016. Knowledge, Skills, and Attributes of Graduate Student and Postdoctoral Mentors in Undergraduate Research Settings. fournal of Engineering Education (2016). https://doi.org/10.1002/jee.20129

[2] Anna N. Baglione, Maxine M. Girard, Meagan Price, James Clawson, and Patrick C. Shih. 2017. Mobile Technologies for Grief Support: Prototyping an Application to Support the Bereaved. In Workshop on Interactive Systems in Health Care (AMIA '17). American Medical Informatics Association.

[3] Anna N. Baglione, Maxine M. Girard, Meagan Price, James Clawson, and Patrick C. Shih. 2018. Modern Bereavement: A Model for Grief and Loss in the Digital Age. In Proceedings of the SIGCHI Conference on Human Factors in Computing Systems (CHI '18). ACM, New York, NY, USA.

[4] Lecia Barker. 2009. Student and Faculty Perceptions of Undergraduate Research Experiences in Computing. ACM Transactions on Computing Education 9, 1 (3 2009), 1-28. https://doi.org/10.1145/1513593.1513598

[5] Gabrielle S. Cantor. 2018. Designing Technological Interventions for Patients with Discordant Chronic Comorbidities and Type-2 Diabetes. In Extended Abstracts of the 2018 CHI Conference on Human Factors in Computing Systems (CHI EA '18). ACM, New York, NY, USA, Article SRC04, 6 pages. https://doi.org/10.1145/ 3170427.3180304

[6] Teresa Dahlberg, Tiffany Barnes, Audrey Rorrer, Eve Powell, and Lauren Cairco. 2008. Improving Retention and Graduate Recruitment Through Immersive Research Experiences for Undergraduates. In Proceedings of the 39th SIGCSE Technical Symposium on Computer Science Education (SIGCSE '08). ACM, New York, NY, USA, 466-470. https://doi.org/10.1145/1352135.1352293

[7] Julia C. Dunbar, Ciabhan L. Connelly, Juan F. Maestre, Haley MacLeod, Katie Siek, and Patrick C. Shih. 2017. Considerations for Using the Asynchronous Remote Communities (ARC) Method in Health Informatics Research. In Workshop on Interactive Systems in Healthcare 2017 at the American Medical Informatics Association Fall Symposium.

[8] Ann Q. Gates. 2008. The Affinity Research Group model : creating and maintaining effective research teams. IEEE Computer Society. 104 pages. https: //www.computer.org/web/cspress/arg

[9] Maria Gorlatova, John Sarik, Peter Kinget, Ioannis Kymissis, and Gil Zussman 2013. Project-based Learning Within a Large-scale Interdisciplinary Research Effort. In Proceedings of the 18th ACM Conference on Innovation and Technology in Computer Science Education (ITiCSE '13). ACM, New York, NY, USA, 207-212. https://doi.org/10.1145/2462476.2465588

[10] Alexander L. Hayes, Mayukh Das, Phillip Odom, and Sriraam Natarajan. 2017. User Friendly Automatic Construction of Background Knowledge: Mode Construction from ER Diagrams. In Knowledge Capture (KCAP) 2017.

[11] Jessica Horowitz and Kelly B. Christopher. 2013. The Research Mentoring Program: Serving the Needs of Graduate and Undergraduate Researchers. Innovative Higher Education 38, 2 (4 2013), 105-116. https://doi.org/10.1007/ s10755-012-9230-3

[12] Ben Jelen, Anne Freeman, Mina Narayanan, Kate M. Sanders, James Clawson, and Katie Siek. 2019. Craftec: Engaging Older Adults in Making through a CraftBased Toolkit System. In Proceedings of the Thirteenth International Conference on Tangible, Embedded, and Embodied Interaction (TEI '19), ACM (Ed.). ACM, New York, NY, USA, 331-340.

[13] Jolene Kay Jesse. 2006. The Poverty of the Pipeline Metaphor: The AAAS/CPST Study of Nontraditional Pathways into IT/CS Education and the Workforce. In Women and Information Technology. The MIT Press, 238-278. https://doi.org/10. 7551/mitpress/9780262033459.003.0008

[14] Karen A. Kim, Amy J. Fann, and Kimberly O. Misa-Escalante. 2011. Engaging Women in Computer Science and Engineering. ACM Transactions on Computing Education 11, 2 (7 2011), 1-19. https://doi.org/10.1145/1993069.1993072

[15] Gloria Ladson-Billings. 2014. Culturally Relevant Pedagogy 2.0: a.k.a. the Remix. Harvard Educational Review (2014). https://doi.org/10.17763/haer.84.1. p2rj131485484751

[16] Haley MacLeod, Grace Bastin, Leslie S. Liu, Katie Siek, and Kay Connelly. 2017. "Be Grateful You Don'T Have a Real Disease": Understanding Rare Disease Relationships. In Proceedings of the 2017 CHI Conference on Human Factors in
Computing Systems (CHI '17). ACM, New York, NY, USA, 1660-1673. https: //doi.org/10.1145/3025453.3025796

[17] Haley MacLeod, Ben Jelen, Annu Prabhakar, Lora Oehlberg, Katie Siek, and Kay Connelly. 2017. A Guide to Using Asynchronous Remote Communities (ARC) for Researching Distributed Populations. EAI Endorsed Transactions, Special Issue on Pervasive Health 2016 (2017).

[18] Juan F. Maestre, Haley MacLeod, Ciabhan L. Connelly, Julia C. Dunbar, Jordan Beck, Katie A. Siek, and Patrick C. Shih. 2018. Defining Through Expansion: Conducting Asynchronous Remote Communities (ARC) Research with Stigmatized Groups. In Proceedings of the 2018 CHI Conference on Human Factors in Computing Systems (CHI '18). ACM, New York, NY, USA, Article 557, 13 pages. https://doi.org/10.1145/3173574.3174131

[19] Jane. Margolis. 2008. Stuck in the shallow end : education, race, and computing. MIT Press. 201 pages.

[20] Jane. Margolis and Allan Fisher. 2002. Unlocking the clubhouse : women in computing. MIT Press. 172 pages.

[21] Tom Ongwere, Gabrielle Cantor, Sergio Ramirez Martin, Patrick C Shih, James Clawson, and Kay Connelly. 2017. Too many conditions, too little time: designing technological intervention for patients with type-2 diabetes and discordant chronic comorbidities. In Workshop on Interactive Systems in Health Care.

[22] Tom Ongwere, Gabrielle Cantor, Ramirez Sergio, Patrick C Shih, James Clawson, and Kay Connelly. 2018. Design Hotspots for Care of Discordant Chronic Comorbidities: Patients' Perspectives. In Proceedings of the Nordic Conference on Human-Computer Interaction. ACM.

[23] John K. Petrella and Alan P. Jung. 2008. Undergraduate Research: Importance, Benefits, and Challenges. International fournal of Exercise Science 1, 3 (2008), 91. https://www.ncbi.nlm.nih.gov/pmc/articles/PMC4739295/

[24] Annu Sible Prabhakar, Lucia Guerra-Reyes, Anne Effron, Vanessa M. Kleinschmidt, Maggie Driscoll, Charles Peters, Vanessa Pereira, Majdah Alshehri, Tom Ongwere, and Katie A. Siek. 2017. "Let Me Know if You Need Anything": Support Realities of New Mothers. In Proceedings of the 11th EAI International Conference on Pervasive Computing Technologies for Healthcare (PervasiveHealth '17). ACM, New York, NY, USA, 31-40. https://doi.org/10.1145/3154862.3154863

[25] Jie Qi, Andrew "bunnie" Huang, and Joseph Paradiso. 2015. Crafting technology with circuit stickers. In Proceedings of the 14th International Conference on Interaction Design and Children - IDC '15. ACM Press, New York, New York, USA, 438-441. https://doi.org/10.1145/2771839.2771873

[26] Daniela Raicu, Audrey Rorrer, and Jamie Payton. 2018. Recruitment, Evaluation, and Tracking. 2018 CISE REU PI Meeting: https://reu.uncc.edu/cise-reutoolkit/results-cise-reu-toolkit.

[27] Olivia K. Richards. 2017. Exploring the Empowerment of Older Adult Creative Groups Using Maker Technology. In Proceedings of the 2017 CHI Conference Extended Abstracts on Human Factors in Computing Systems (CHI EA '17). ACM, New York, NY, USA, 166-171. https://doi.org/10.1145/3027063.3048425

[28] Audrey Rorrer. [n. d.]. Evaluation Plans: Reserach Experience for Undergraduates: Socially Relevant Computing. http://reu.uncc.edu/toolkit/assessment/evaluationplans.

[29] Audrey Smith Rorrer, Joseph Allen, and Huifang Zuo. 2018. A National Study of Undergraduate Research Experiences in Computing. In Proceedings of the 49th ACM Technical Symposium on Computer Science Education - SIGCSE '18. ACM Press, New York, New York, USA, 604-609. https://doi.org/10.1145/3159450. 3159510

[30] Jeffery R. Schultz. 2001. The Transformational Process of Mentoring. In Council on Undergraduate Research Quarterly. Vol. 22. Council on Undergraduate Research, $72-73$.

[31] Patricia J. Teller and Ann Q. Gates. 2001. Using the Affinity Research Group Model to Involve Undergraduate Students in Computer Science Research*. Fournal of Engineering Education 90, 4 (10 2001), 549-555. https://doi.org/10.1002/j. 2168-9830.2001.tb00638.x

[32] Elsa Q. Villa, Kerrie Kephart, Ann Q. Gates, Heather Thiry, and Sarah Hug. 2013. Affinity Research Groups in Practice: Apprenticing Students in Research. Fournal of Engineering Education 102, 3 (7 2013), 444-466. https://doi.org/10.1002/jee. 20016 